\title{
Reflets
}

Revue ontaroise d'intervention sociale et communautaire

\section{Le travail social auprès des personnes âgées en Ontario}

\section{Richard Carrière}

Volume 7, numéro 2, automne 2001

Le travail social en Ontario

URI : https://id.erudit.org/iderudit/026358ar

DOI : https://doi.org/10.7202/026358ar

Aller au sommaire du numéro

Éditeur(s)

Reflets : Revue ontaroise d'intervention sociale et communautaire

ISSN

1203-4576 (imprimé)

1712-8498 (numérique)

Découvrir la revue

Citer cet article

Carrière, R. (2001). Le travail social auprès des personnes âgées en Ontario. Reflets, 7(2), 104-117. https://doi.org/10.7202/026358ar

Tous droits réservés (C) Reflets : Revue ontaroise d'intervention sociale et communautaire, 2001
Ce document est protégé par la loi sur le droit d'auteur. L'utilisation des services d'Érudit (y compris la reproduction) est assujettie à sa politique d'utilisation que vous pouvez consulter en ligne.

https://apropos.erudit.org/fr/usagers/politique-dutilisation/ 


\section{Le travail social auprès des personnes âgées en Ontario}

par

Richard Carrière, professeur, École de service social, Université Laurentienne

Le but de cet article est de brosser un tableau du travail social auprès des personnes âgées ${ }^{1}$ en Ontario et de partager nos réflexions sur ce champ de pratique. Nous débuterons l'article en nous rappelant l'origine du travail social auprès des personnes âgées et en soulignant les tendances qui ont marqué son développement. Par la suite, nous identifierons sommairement les divers milieux de pratique des travailleuses sociales et des travailleurs sociaux, appelés à aider les personnes âgées. Enfin, nous poserons un regard sur l'avenir de ce secteur du travail social.

\section{Survol historique}

La profession du travail social est née à une époque où la population était plus jeune qu'aujourd'hui. Selon les données de Statistique Canada, en 1901, seulement 5,1\% de la population était âgée de 65 ans et plus, alors qu'en 2001, on estime que cette proportion s'élève à 12,9\% (Desjardins 1993). La profession du travail social a donc vu le jour à une époque où les taux de natalité étaient plus élevés qu'aujourd'hui et où l'espérance de vie y était nettement inférieure. 
Si l'espace ne nous permet pas ici de tracer à fond l'évolution historique du service social en Ontario, il importe de souligner qu'au début de la profession deux clientèles y ont occupé une place importante. D'une part, le mouvement en faveur de la protection des enfants a mené à la création des Sociétés d'aide à l'enfance et des autres services pour les jeunes et leur famille. D'autre part, le développement des services a permis de répondre aux besoins des nouveaux immigrés dans les grands centres urbains.

Le travail social en protection de la jeunesse est davantage une intervention clinique, alors que le travail auprès des nouveaux immigrés est davantage une intervention communautaire, axée sur les problèmes de l'urbanisation et de l'industrialisation auxquels faisaient face une population adulte. Ainsi, les personnes âgées figurent peu dans les services sociaux offerts à la population au début du vingtième siècle. Ce n'est pas qu'elles en soient exclues, mais elles ne sont pas encore perçues comme une clientèle suffisamment importante pour recevoir des services spécifiques.

Comme l'explique Holosko:

Depuis le début de la pratique du service social [...] la profession a généralement agi et pris soin des personnes âgées de la même façon que l'a fait la société en général. Les gouvernements et les structures sociopolitiques $d u$ monde occidental ont généralement supposé que les institutions et les mécanismes principaux de bien-être social, par exemple l'Église, l'État, le régime de soins de santé, les agences sociales et familiales, les programmes et politiques sociales, etc., assumaient les soins des personnes âgées (1996: 22)2.

Selon Holosko, le développement des services sociaux pour les personnes âgées a été plutôt réactif que pro-actif. On répondait davantage aux problèmes que chercher à les prévenir. De plus, le travail social auprès des personnes âgées a suivi l'émergence des divers programmes destinés à répondre, de prime abord, aux besoins médicaux des personnes âgées. Dès le début des années 1900 , on trouve dans certains grands centres urbains comme 
Boston, des travailleurs sociaux qui aident les patients dans les hôpitaux (Netting 1990). On peut assumer qu'une portion de ces patients étaient des personnes âgées. Aujourd'hui, avec le vieillissement de la population, les personnes âgées occupent de plus en plus de lits dans les hôpitaux. En effet, plus une personne vieillit, plus elle est susceptible de recourir aux services de santé et, plus elle est susceptible d'être hospitalisée. Ceci ne veut pas dire que toute personne âgée a des besoins exigeant des services hospitaliers, mais les risques d'y avoir recours augmentent avec l'âge. Comme l'explique Dumas, «le vieillissement de l'organisme engendre une détérioration de la santé qui se traduit par la maladie, des limitations de l'activité, l'invalidité, avec en conséquence le recours aux hôpitaux» (1993: 101). Au cours de l'Enquête sociale générale, menée par Statistique Canada en 1986,18 \% des personnes âgées de 65 ans et plus ont été hospitalisés. Par ailleurs, «il est important de souligner qu'un grand nombre de personnes âgées demeurent autonomes dans leur fonctionnement en dépit de problèmes de santé» (Beaudoin 1988: 89).Ainsi, il n'est pas étonnant de constater que la majorité des personnes âgées, tout âge confondu, jugent leur état de santé bon ou excellent et que seule une personne sur dix le juge tout à fait mauvais (Dumas 1993).

Holosko explique le développement des services aux personnes âgées à partir d'une perspective inter-organisationnelle. Il soutient que les foyers pour personnes âgées ont été créés pour répondre à une hospitalisation grandissante des personnes âgées durant les années 1950 et 1960 :

lorsque les foyers pour personnes âgées ne pouvaient plus prendre soin convenablement des personnes âgées frêles qui vivaient plus longtemps qu'auparavant, des institutions de soins chroniques ou des aménagements à l'intérieur d'une institution ont surgi vers la fin des années 1970. Lorsque les coûts de ces dernières sont devenus trop dispendieux, on s'est tourné vers des pourvoyeurs de soins communautaires et des programmes de répit avec des aidants naturels au début des années 1980 pour économiser (Holosko 1996: 23). 
Même si l'on assiste progressivement à la mise en place d'une variété de services pour répondre aux besoins de santé, d'hébergement ou de soutien des personnes âgées, il ne faut pas oublier que la responsabilité de l'assistance aux personnes âgées incombait à la famille. La famille a été et continue d'être la principale institution sociale répondant aux besoins des personnes âgées lorsque celles-ci ne sont pas en mesure d'y répondre personnellement. La famille est présente dans toute personne qui vieillit. Pourtant, la famille d'aujourd'hui est bien différente de la famille traditionnelle et l'explosion démographique contemporaine a eu un tel effet sur la famille qu'on ne peut pas comparer ce que la famille fait aujourd'hui pour ses parents âgés avec ce qu'elle faisait il y a cent ans. Cela dit, «la famille d'aujourd'hui s'occupe de ses parents âgés autant qu'autrefois, peut être même plus» (Laforest 1997:24). Avec le vieillissement de notre population, nous sommes devenus, selon Renée Solomon, «la première génération de l'histoire dans laquelle beaucoup de personnes qui ont 40,50 et 60 ans sont en même temps enfants, époux, parents et grandsparents» (cité dans Getzel et Millor 1982: 84). Il faut souligner cependant que dans les familles, ce sont principalement «les femmes qui ont tendance à assumer le rôle de fournisseur de soins, en plus de leurs responsabilités familiales, professionnelles et communautaires» (Ontario 1991: 4). Ainsi, les femmes de nos jours sont souvent prises avec les responsabilités de leurs enfants, de leur conjoint, de leur ménage, de leur emploi et de leurs parents vieillissants. Elles ont besoin d'aide pour assumer ces diverses responsabilités.

Bien qu'il y ait des services et des programmes créés pour aider les personnes âgées et leur famille, il faut souligner que les services sociaux et le rôle des travailleurs sociaux different d'une province à l'autre au Canada, compte tenu qu'il s'agit d'un champ de compétence provincial ou territorial. Dans certaines provinces comme au Québec, les travailleurs sociaux et les travailleuses sociales sont partie intégrante de certains services, comme c'est le cas dans les établissements de soins à longue durée. En Ontario, la situation differe. Par exemple, parmi les quatre établissements de soins de longue durée à Sudbury, située dans le Nord-Est de la 
province, seuls deux d'entre eux offrent les services d'une travailleuse sociale. Dans certains grandes agglomérations urbaines comme Toronto, il y a des centres comme le Baycrest Centre, où les travailleuses sociales et les travailleurs sociaux occupent une place importante au sein d'équipes multidisciplinaires. Ainsi, la présence des travailleuses sociales et des travailleurs sociaux dans les divers établissements desservant les personnes âgées dépend des régions de la province ${ }^{3}$.

Jusqu'à récemment, en Ontario, les services de soins de longue durée et les services de soutien aux personnes étaient fragmentés. De plus,l'accessibilité aux services sur le territoire provincial n'était pas garantie, les services étant inégalement répartis (Ontario 1991). Pour combler cette lacune, le gouvernement de l'Ontario a mis sur pied quarante-trois (43) centres d'accès aux soins communautaires. Ces centres ont pour mandat de coordonner, à l'échelle communautaire, la prestation des services de santé et des soins à domicile, en plus de gérer la transition des soins entre l'hôpital et le domicile des patients ou l'admission aux centres de soins de longue durée. En théorie, des travailleuses sociales et des travailleurs sociaux devaient être une composante du personnel de ces centres. Même la documentation de l'Association des travailleuses et des travailleurs sociaux de l'Ontario fait l'éloge du fait que «le service social est un élément clé du système de soins de longue durée» (ATTSO 1994:9). Pourtant, leur rôle ne nous apparaît pas central jusqu'à présent, car ce sont surtout les infirmières qui assument les tâches de gestion de cas, en faisant l'évaluation des besoins et de la capacité des clients et en assurant la coordination des services des placements. Les travailleuses et les travailleurs sociaux, comme d'ailleurs les ergothérapeutes, les physiothérapeutes, les orthophonistes ou les autres professionnels de la santé jouent, dans ces centres, un rôle complémentaire en offrant certains services spécifiques. De plus, il arrive souvent que ces services soient offerts en sous-traitance par des intervenantes et des intervenants travaillant à leur propre compte ou pour des compagnies privées.

Avant les années 1970, la profession du service social a porté peu d'attention clinique, théorique ou empirique sur la pratique du service social auprès des personnes âgées, ce qui explique le 
peu de présence des travailleuses sociales et des travailleurs sociaux en Ontario dans ce domaine. Ce n'est qu'à partir de 1980 que la profession du service social commence, selon Holosko (1996), à reconnaitre les besoins spécifiques des personnes âgées et à élaborer des programmes spécialisés de formation en travail social auprès des personnes âgées. À la même époque, en 1978, la création d'une revue portant sur Gerontological Social Work, publié par Haworth Press, témoigne de l'intérêt croissant pour ce champ de pratique. De plus, les institutions d'éducation postsecondaire commencent à développer des cours et même des certificats d'études sur le travail social auprès des personnes âgées. Plusieurs documents de planification des services pour les personnes âgées laissent croire à une participation active de la profession du service social. Or, la majorité de ces services offerts aux personnes âgées relèvent du Ministère de la santé et non, du Ministère des Services sociaux et communautaires. Dans un tel contexte, le travail social auprès des personnes âgées se pratique surtout sous forme de collaboration interprofessionnelle où domine l'aspect médical.

Le travail social est une discipline «où la formation, l'éducation, les principes de bases et les activités professionnelles visent spécifiquement à tirer le maximum des points forts ou des ressources des individus et à supprimer les obstacles qui existent entre eux et les services ou les programmes dont ils ont besoin» (ATTSO 1994: 9). Cela dit, comme l'explique Sheila Neysmith, «la fonction de l'organisme définit, en grande partie, le rôle des travailleuses et des travailleurs sociaux" (traduction libre, 1988: 50). En Ontario, il y a trois principaux milieux qui embauchent des travailleuses sociales et des travailleurs sociaux pour aider les personnes âgées. Ces milieux sont les hôpitaux, les centres communautaires et les services de soins à longue à durée. De plus, la gamme de services sociaux disponibles à la population adulte de la province est aussi disponible aux personnes âgées. Pensons, en autres, aux services de l'Institut national canadien pour les aveugles ou aux services de la Société canadienne de l'ouie. De plus, les organismes comme les Services familiaux peuvent offrir de la thérapie familiale et conjugale à la fois aux jeunes familles et aux «vieilles familles ou aux vieux couples». De 
plus en plus, les Conseils de planification sociale se préoccupent des besoins des personnes âgées dans leur communauté. Toutefois, en Ontario, il n'y a pas, comme aux États Unis, d'organisme de protection des personnes âgées.

En Ontario et ailleurs au Canada, dans les divers débats consacrés au problème de la violence infligée aux personnes âgées, les personnes âgées n'ont pas voulu la création d'un système comparable à celui de la protection de la jeunesse. Les personnes âgées ne veulent pas d'un service infantilisant. Elles préconisent que la violence à leur égard doit être traitée comme un crime et qu'à ce titre, l'intervention devrait viser l'agresseur et non pas, chercher à leur enlever leur autonomie (MacClean 1995). Ainsi, il existe divers services sociaux et communautaires où les personnes âgées peuvent profiter, selon les circonstances, du professionnalisme d'une travailleuse sociale ou d'un travailleur social. Par ailleurs, dans certains milieux, les travailleuses sociales ou les travailleurs sociaux sont embauchés spécifiquement pour venir en aide aux besoins liés au vieillissement de la personne.

\section{Principaux milieux d'intervention des travailleuses sociales et des travailleurs sociaux}

\section{Les hôpitaux}

Selon Sheila Neysmith (1988), le milieu d'intervention où on trouve le plus grand nombre de travailleuses sociales et de travailleurs sociaux œuvrant auprès des personnes âgées est le milieu hospitalier. Comme l'explique Ellen Netty (1990), deux objectifs orientent la pratique du travail social en milieu hospitalier. Dans un premier temps, la travailleuse sociale ou le travailleur social aide le patient et sa famille à s'adapter au traumatisme et au stress, suscités par l'hospitalisation. Dans un deuxième temps, la travailleuse sociale ou le travailleur social appuie le patient dans sa prise de décision afin que ce dernier garde le contrôle avec tout ce qui se passe autour de lui. 
Susan Blumenfield dit que :

La pratique du travail social avec les gens plus âgés à l'hôpital n'inclut pas seulement le patient, mais aussi sa famille ou les autres personnes significatives. Les travailleurs sociaux de l'hôpital travaillent avec les autres intervenants de l'hôpital de plusieurs façons, mais aussi avec le personnel des agences communautaires, des institutions de soins de longue durée, des organismes de politiques sociales et des groupes de défense des droits des personnes âgées. Le travailleur social à l'hôpital doit souvent coordonner des services à l'intérieur et à l'extérieur de l'institution et doit aider à équilibrer les besoins de plusieurs personnes qui interviennent auprès du patient. [...] La pratique $d u$ travail social à l'hôpital se fait généralement dans des conditions de crise et des limites de temps restreintes [...] beaucoup doit être fait dans de brefs délais (Blumenfield cité dans Getzel et Millor 1982: 38).

Actuellement, les travailleuses sociales et les travailleurs sociaux des hôpitaux que nous avons côtoyés, disent qu'ils ne font que planifier l'accueil et le départ de l'hôpital. Comme les séjours d'hospitalisation sont de plus en plus courts, ils n'ont pas le temps de s'attaquer aux vrais problèmes. Le travail social en milieu hospitalier ne se limite pas aux services de soins aigus physiques. Dans certaines régions de l'Ontario, les hôpitaux offrent aussi un programme de soins psychogériatriques afin de fournir un diagnostic et un traitement aux personnes âgées qui souffrent de problèmes émotifs ou psychologiques. Les travailleuses sociales et les travailleurs sociaux font généralement partie de ces équipes d'intervention.

\section{Les programmes de soutien communautaire}

Le deuxième plus important milieu de travail pour les travailleuses sociales et les travailleurs sociaux œuvrant auprès des personnes âgées en Ontario est relié aux programmes de soutien communautaire. Dans un document publié par l'Association des 
travailleuses et travailleurs sociaux de l'Ontario, les services de travail social offerts dans les centres de soutien communautaire sont liés aux besoins et conditions suivantes.

- Vérifier les ressources: évaluer la condition de vie actuelle du client et ses besoins; identifier les choix; aiguiller les personnes vers les spécialistes; recommander la livraison appropriée des services et programmes surtout quand ceux-ci sont compliqués et font l'objet de demandes spéciales.

- L'adaptation à la maladie ou l'incapacité: renforcer les capacités de faire face aux sentiments de perte, de chagrin et du changement de rôle; évaluer et intervenir face aux comportements d'anxiété, de dépression ou de mésadaptation sociale; aider à la reconnaissance de l'impact de la maladie ou l'incapacité sur les relations de familles; faciliter les liens entre les divers réseaux d'appui.

- Question de placement: aider le client et sa famille à comprendre les nécessités des soins; les aider à participer et prendre part aux décisions les concernant et les préparer aux émotions de la transition.

- Abus et négligence: évaluer les risques; informer sur les conséquences des choix orientés vers le changement; offrir de la thérapie pour réduire ou éliminer les risques et garantir la sécurité; faciliter les demandes de consultation pour les questions légales, financières, de logement ou autres.

- Soins palliatifs: aider le client et sa famille à comprendre la nécessité des soins palliatifs et les choix possibles; promouvoir la communication ouverte et des stratégies positives pour permettre une prise de décision efficace; fournir de l'information et aiguiller les personnes vers les ressources communautaires; offrir un suivi.

- Surmenage (burnout) du pourvoyeur des soins: faciliter l'identification des facteurs de stress et des stratégies d'adaptation; fournir l'information et l'appui aux pourvoyeurs de soins en ce qui concerne des questions telles l'isolement, les changements de rôle; établir les liens avec les ressources communautaires. 
- Isolement social: évaluer si le soutien social existant est adéquat; explorer les possibilités avec le client et sa famille afin de fortifier l'appui formel et informel; intervenir pour réduire les obstacles à l'interaction.

- Conflits familiaux ou intergénérationnels: évaluer et fournir de la thérapie psychoéducationnelle, par exemple, pour surmonter les problèmes de communication, d'interaction sociale, de dysfonctionnement et des situations stressantes de comportements qui peuvent avoir précédé les problèmes de santé; aiguiller les personnes vers les services de santé mentale.

- Problèmes psychosociaux individuels: évaluer le rôle des facteurs émotionnels et sociaux sur la situation de la santé et le comportement, par exemple l'anorexie, l'insomnie; fournir de la thérapie psychoéducationnelle pour aider le client à reconnaitre les effets de ses comportements sur son bien-être (ATTSO 2001, notre traduction).

\section{Les centres de santé communautaire}

En plus des centres d'accès aux soins communautaires, qui jouent davantage un rôle d'aiguillage aux services communautaires, il y a en Ontario cinquante-quatre (54) centres de santé communautaire qui, tout en offrant des services de santé primaire, cherchent à mettre l'accent sur la promotion de la santé,favorisant ainsi la prévention des problèmes de la santé.

Certains centres comme celui de Sudbury ont spécifiquement identifié les personnes âgées comme faisant partie de leurs clientèles-cibles. Bien qu'il y ait une travailleuse sociale au Centre de santé communautaire de Sudbury, le travail auprès des personnes âgées relève davantage de l'intervenante en promotion de la santé. Dans certains cas, cette dernière peut avoir une formation en service social, mais celle-ci n'est pas une exigence pour le poste. Le travail de promotion de la santé est axé sur l'entraide, l'autonomie et l'indépendance des personnes âgées. En certaines circonstances comme celles qui prévalent à Sudbury, les intervenantes de la promotion de la santé vont travailler de près avec les clubs d'âge d'or afin de rendre plus accessible leur programmation et d'appuyer les projets des divers clubs. 


\section{Les établissements de soins de longue durée}

Bien que les services de maintien à domicile soient privilégiés pour certaines personnes âgées, il n'est pas toujours possible de répondre adéquatement à leurs besoins dans le propre logement. En dépit des services de soutien à domicile offerts par la famille ou par les divers organismes communautaires, il arrive que la perte d'autonomie soit tellement grande que la personne âgée n'a pas d'autres choix que de recourir aux services d'hébergement de soins de longue durée ou des foyers pour personnes âgées. D'ailleurs, aujourd'hui, le critère d'accessibilité à l'hébergement ou aux foyers, est la perte d'autonomie.Ainsi, «les centres d'accueil, d'hébergement ainsi que les centres hospitaliers de soins prolongés, sont des milieux de vie substituts qu'on dit lourds" (Beaudoin 1988: 940). Ceux et celles qui jouissent toujours de leur autonomie doivent se tourner vers des établissements privés. Notons que ces foyers sont très dispendieux et sont souvent éloignés du lieu de résidence de la famille immédiate.

La présence des travailleuses sociales et des travailleurs sociaux dans le secteur de service de soins de longue durée «est assez faible» (Neysmith 1988:51). Une fois encore, la taille du centre et de la communauté est directement reliée à la présence ou non de travailleuses sociales et de travailleurs sociaux. De plus, les tâches professionnelles exigées varient d'un établissement à l'autre.

«Dans les foyers pour personnes âgées, la tâche centrale du travailleur social était de faciliter l'adaptation du client à l'institution" (Silverstone et Burack-Weiss 1982: 7). Par contre, Greene et ses collègues suggèrent que:

Peut-être que le facteur le plus important portant sur la pratique actuelle du travail social dans les foyers de personnes âgées est l'introduction de nouveaux règlements et de nouvelles exigences législatives mettant davantage l'accent sur la protection de la qualité de vie du résident. [...] Ce renouveau d'intérêt dans les soins psychosociaux vient renforcer les services $d u$ travail social dans les foyers de personnes âgées (1992: 40). 


\section{Avenir du travail social auprès des personnes âgées}

Il est estimé que les personnes âgées de 65 ans et plus au Canada formeront, d'ici l'an 2036, de 20 à $25 \%$ de l'ensemble de la population. De plus,

les personnes de plus de 75 ans formeront sans doute plus de la moitié du groupe des 65 ans et plus, alors qu'elles en formaient que $30 \%$ en 1950. Quant aux grands vieillards (85 ans et plus), durant cette période, ils verront leur nombre triplé. Ce vieillissement du vieillissement ne peut qu'exacerber les conséquences socio-économiques du phénomène lui-même (Dumas 1993: 1).

Kosberg suggère que «la population vieillissante $[\ldots]$ offre un terrain aux mille ramifications pour la participation possible des travailleurs sociaux, des chercheurs et des éducateurs» (1999: 8). Il ajoute que «si les travailleurs sociaux ne prennent pas avantage des opportunités de carrières en gérontologie, ils vont perdre leur place au détriment d'intervenants provenant de d'autres professions» (Kosberg 1999: 7).

Jusqu'à présent, «le travail social s'attarde beaucoup plus aux conséquences problématiques qui émergent du vieillissement des populations et des individus, puisque l'objet et l'expertise de la profession du travail social sont les problèmes sociaux» (Ladouceur 1996: 93). Mais l'intervention devrait aller au-delà des problèmes d'hébergement et des soins de longue durée. Comme le suggère Jean Carette, la travailleuse sociale ou le travailleur social, n'est pas seulement un agent d'aide :

[elle ou il] est avant tout un agent facilitant et suscitant le changement social: ce qui fait toute la différence avec un simple informateur, avec un animateur ou encore, avec un conseiller psychologique. [La travailleuse sociale 
ou le travailleur social] se doit de travailler le social, c'est-à-dire d'inscrire ses interprétations, ses actions, ses médiations dans une perspective de changement institutionnel [...] Les problèmes d'un individu demandeur ne sont à cet égard que le symptôme d'un problème social, c'est-à-dire de portée générale et remettant en cause l'ordre établi (1996, Module VIII: 2).

Face à une société qu'elle qualifie de capitaliste, âgiste et patriarcale, Ladouceur invite les travailleuses sociales et les travailleurs sociaux à «jouer un rôle de dénonciateur des discriminations» auxquelles les personnes âgées sont confrontées (1996: 95). Mais, on ne peut limiter nos actions à la dénonciation, nous devons axer notre pratique sur l'intervention sociale qui «vise la transformation et l'amélioration d'une situation jugée et vécue comme préjudiciable à l'individu et au groupe» (Carette 1996, Module VII: 3).

\section{Bibliographie}

BEAUDOIN,A. (1988). Services sociaux à l'enfance, à la famille, aux personnes âgées et dans le domaine de la santé. L'État de la situation au Québec, Québec, Laboratoire de Recherche, École de service social, Université Laval.

BLUMMENFIELD, S. (1982). «The Hospital Center and Aging:A Challenge for the Social Worker», dans G. Getzel et J. Mellor (Eds.), Gerontological Social Work Practice in Long-Term Care, New York: Haworth Press, 35-60.

CARETTE, J. (1992). Manuel de gérontologie sociale 1, Boucherville. Gaëtan Morin éditeur.

CARETTE, J. (1996). SESO 4446 : Le service social auprès des personnes agées. Sudbury, Centre d'éducation permanente, Université Laurentienne.

GETZEL, G. et J. Mellor (Eds.) (1982). Gerontological Social Work Practice in Long-Term Care, New York: Haworth Press.

GREENE, R., B.VOURLEKIS, D. GELFAND et J. LEWIS (1992). «Current Realities: Practice and Education Needs of Social Workers in Nursing Homes», Journal of Gerontological Social Work, vol. 18 , nos 3 et 4, 39-54.

HOKENSTAD, M. et K. KENDALL (Eds.) (1988). Gerontological Social Work: International Perspectives, New York, Haworth Press.

HOLOSKO, M. et M. FEIT (Eds.) (1996). Social Work Practice with the Elderly (2nd ed.), Toronto, Canadian Scholars' Press. 
LADOUCEUR, L. (1996). «Différences chez les personnes âgées: mise en contexte», Reflets: Revue d'intervention sociale et communautaire, vol.2 no 2, 82-99.

LAFOREST, J. (1997). Gérontologie appliquée: les professionnelles de la vieillesse, Montréal, HMH.

MACLEAN, M. (1995). Mauvais traitements auprès des personnes âgées: stratégies de changement, Montréal, Éditions Saint-Martin.

NETTING, E. (1990). «Hospital Based Long Term Care Services for the Frail Elderly», Journal of Gerontological Social Work, vol. 15, nos 3-4, 33-48.

NEYSMITH, S. (1988). "Canadian Social Services and Social Work Practice in the Field of Aging», dans M. Hokenstad et K. Kendall (Eds.), Gerontological Social Work: International Perspectives, New York, Haworth Press, 41-60.

ONTARIO ASSOCIATION OF SOCIAL WORKERS (2001). Community-Based Health Care: Social Work Services. Toronto, OASW.

ROWLINGS, C. (1981). Social Work with Elderly People, London, George Allen \& Unwin.

SOLOMON, R. (1982). «Serving Families of the Institutionalized Aged:The Four Crises», dans G. Getzel et J. Mellor (Eds.), Gerontological Social Work Practice in Long Term Care, New York, Haworth Press, 83-96.

\section{Notes}

1. Pour les fins de cet article, une personne âgée est celle qui a 65 ans et plus. Parmi ce groupe de personnes, certains font une distinction entre les jeunes vieux ( 65 ans à 74 ans), les moyens vieux (75 ans à 84 ans) et les vieux (85 ans et plus). Nous pourrions même ajouter la catégorie des centenaires, car il y a de plus en plus de personnes qui atteignent et dépassent les cent ans.

2. Les traductions des citations d'auteurs anglais sont des traductions libres.

3. Il serait intéressant de mener une étude provinciale afin d'identifier la présence des travailleuses sociales et des travailleurs sociaux oeuvrant auprès des personnes âgées. 